\title{
Large $q$ expansion in the Sachdev-Ye-Kitaev model
}

\author{
Grigory Tarnopolsky \\ Department of Physics, Harvard University, Cambridge, Massachusetts 02138, USA
}

(Received 20 September 2018; published 15 January 2019)

\begin{abstract}
We consider the Sachdev-Ye-Kitaev (SYK) model, where interaction involves $q$ fermions at a time. We find the $1 / q^{2}$ correction to the thermal two-point function in the large $q$ expansion. Using this result, we find the $1 / q^{3}$ correction to the SYK free energy.
\end{abstract}

DOI: 10.1103/PhysRevD.99.026010

\section{INTRODUCTION}

The Sachdev-Ye-Kitaev (SYK) model is a quantum mechanical model of $N$ interacting Majorana fermions $\chi_{i}, i=1, \ldots, N$ with the Hamiltonian $[1,2]$,

$$
H_{\mathrm{SYK}_{q}}=(i)^{\frac{q}{2}} \sum_{1 \leq i_{1}<i_{2}<\ldots<i_{q} \leq N} J_{i_{1} \ldots i_{q}} \chi_{i_{1}} \chi_{i_{2}} \ldots \chi_{i_{q}},
$$

where $\left\{\chi_{i}, \chi_{j}\right\}=\delta_{i j}$ and $J_{i_{1} \ldots i_{q}}$ are random couplings drawn from a Gaussian distribution with zero mean and a width $\left\langle J_{i_{1} \ldots i_{q}}^{2}\right\rangle=(q-1) ! J^{2} / N^{q-1}$. One is usually interested in computing correlation functions and, particularly, the two-point function at temperature $T=1 / \beta$ :

$$
G(\tau)=\langle\mathrm{T} \chi(\tau) \chi(0)\rangle_{\beta}
$$

At the large $N$ limit, only melonic Feynman diagrams contribute to the two-point function in the SYK model. These diagrams can be resummed and one obtains a nonperturbative Schwinger-Dyson equation,

$G\left(i \omega_{n}\right)^{-1}=-i \omega_{n}-\Sigma\left(i \omega_{n}\right), \quad \Sigma(\tau)=J^{2} G(\tau)^{q-1}$

where $G\left(i \omega_{n}\right)=\int_{0}^{\beta} d \tau e^{i \omega_{n} \tau} G(\tau)$ and $\omega_{n}=2 \pi \beta^{-1}(n+1 / 2)$. It is not possible to solve this equation analytically, but one can find solution in the infrared limit, where $\omega$ is small and the bare $-i \omega_{n}$-term in (3) can be neglected [1-4],

$$
G_{c}(\tau)=b\left(\frac{\pi}{\beta \sin \frac{\pi \tau}{\beta}}\right)^{2 / q} \operatorname{sgn}(\tau)
$$

Published by the American Physical Society under the terms of the Creative Commons Attribution 4.0 International license. Further distribution of this work must maintain attribution to the author(s) and the published article's title, journal citation, and DOI. Funded by SCOAP ${ }^{3}$. where $J^{2} b^{q} \pi=(1 / 2-1 / q) \tan (\pi / q)$. Nevertheless, it is still interesting to obtain some analytic approximation for $G(\tau)$ which interpolates both UV and IR regions. One way to proceed is to use the large $q$ expansion. The first order in $1 / q$ was found in [3]. In this article, we compute the next $1 / q^{2}$ correction and argue that it improves the approximation significantly, such that it agrees with numerical results quite well.

In the next section, we compute the $1 / q^{2}$ correction to the two-point function. Next, we compare the large $q$ results and numerics. At the end, we compute the large $q$ free energy and the coefficient of the Schwarzian action.

\section{LARGE $q$ TWO-POINT FUNCTION}

We consider the large $q$ ansatz for the two-point function [3]:

$$
G(\tau)=\frac{1}{2} \operatorname{sgn}(\tau)\left(1+\frac{1}{q} g(\tau)+\frac{1}{q^{2}} h(\tau)+\cdots\right) .
$$

For the self-energy (3), we find (we assume that $q$ is even)

$\Sigma(\tau)=\frac{\mathcal{J}^{2}}{q} \operatorname{sgn}(\tau) e^{g}\left(1+\frac{1}{q}\left(h-g-\frac{1}{2} g^{2}\right)+\ldots\right)$,

where a new coupling constant $\mathcal{J}^{2}=2^{1-q} q J^{2}$ is introduced. From now on, we work on the interval $\tau \in[0, \beta]$ and we can omit $\operatorname{sgn}(\tau)$ in all formulas. Expanding $G\left(i \omega_{n}\right)^{-1}$ in the $1 / q$ series up to the $1 / q^{2}$ term using (5), we obtain

$$
\begin{aligned}
G\left(i \omega_{n}\right)^{-1}= & -i \omega_{n}+\frac{1}{2 q} \omega_{n}^{2} g\left(i \omega_{n}\right) \\
& +\frac{\omega_{n}^{2}}{2 q^{2}}\left(h\left(i \omega_{n}\right)+\frac{i \omega_{n}}{2} g * g\left(i \omega_{n}\right)\right),
\end{aligned}
$$

where $g * g\left(i \omega_{n}\right) \equiv \int_{0}^{\beta} d \tau e^{i \omega_{n} \tau} g^{2}(\tau)$. Then using Eqs. (3) and (6) and going back to the coordinate space, we find differential equations for each order of $1 / q$, 
$\partial_{\tau}^{2} g=2 \mathcal{J}^{2} e^{g}$,

$\partial_{\tau}^{2} h=2 \mathcal{J}^{2} e^{g} h+\frac{1}{2} \partial_{\tau}^{3}(g * g)-2 \mathcal{J}^{2} e^{g}\left(g+\frac{1}{2} g^{2}\right)$,

and the functions $g(\tau)$ and $h(\tau)$ satisfy the boundary conditions $g(0)=g(\beta)=0$ and $h(0)=h(\beta)=0$. Now we introduce a convenient variable $x=\frac{\pi v}{2}-\frac{\pi v \tau}{\beta}$. Then the first equation has the solution

$$
g(x)=\log \left(\frac{\cos \frac{\pi v}{2}}{\cos x}\right)^{2}, \quad \beta \mathcal{J}=\frac{\pi v}{\cos \frac{\pi v}{2}} .
$$

Using this solution, the second equation can be represented as

$$
\begin{aligned}
& \left(\partial_{x}^{2}-\frac{2}{\cos ^{2} x}\right) h(x) \\
& \quad=-\frac{\pi v}{\beta} \frac{1}{2} \partial_{x}^{3}(g * g)-\partial_{x}^{2} g(x)\left(g(x)+\frac{1}{2} g^{2}(x)\right) .
\end{aligned}
$$

The solution to this equation can be written as

$$
\begin{aligned}
h(x)= & -\int_{-\frac{\pi v}{2}}^{\frac{\pi v}{2}} d y \mathcal{G}(x, y)\left(\frac{\pi v}{\beta} \frac{1}{2} \partial_{y}^{3}(g * g)\right. \\
& \left.+\partial_{y}^{2} g(y)\left(g(y)+\frac{1}{2} g^{2}(y)\right)\right),
\end{aligned}
$$

where the Green's function $\mathcal{G}(x, y)$ obeys the equation

$$
\left(\partial_{x}^{2}-\frac{2}{\cos ^{2} x}\right) \mathcal{G}(x, y)=\delta(x-y)
$$

with the boundary conditions $\mathcal{G}\left(-\frac{\pi v}{2}, y\right)=\mathcal{G}\left(\frac{\pi v}{2}, y\right)=0$. One can solve this equation and obtain an explicit formula for the Green's function,

$\mathcal{G}(x, y)=\frac{1}{2 V}\left(\left(V+x_{<}\right) \tan x_{<}+1\right)\left(\left(V-x_{>}\right) \tan x_{>}-1\right)$,

where $V \equiv \frac{\pi v}{2}+\cot \frac{\pi v}{2}$ and $x_{>} \equiv \max (x, y)$ and $x_{<} \equiv$ $\min (x, y)$. Computing the convolution

$$
\begin{aligned}
& \frac{\pi v}{\beta} \frac{1}{2} \partial_{x}^{3}(g * g) \\
& \quad=2 \partial_{x}\left(g(x)\left(\cot \left(\frac{\pi v}{2}+x\right)-\cot \left(\frac{\pi v}{2}-x\right)\right)\right)-4
\end{aligned}
$$

and using the explicit formula for the Green's function (13), we obtain from (11)

$$
\begin{aligned}
h(x)= & \frac{1}{2} g^{2}(x)-2 \ell(x)-4\left(\tan x \int_{0}^{x} d y \ell(y)+1\right) \\
& +4 \frac{1+x \tan x}{1+\frac{\pi v}{2} \tan \frac{\pi v}{2}}\left(\tan \frac{\pi v}{2} \int_{0}^{\frac{\pi v}{2}} d y \ell(y)+1\right),
\end{aligned}
$$

where $\ell(x) \equiv g(x)-e^{-g(x)} \operatorname{Li}_{2}\left(1-e^{g(x)}\right)$ and $g(x)$ is given in (9). One can compute explicitly the integral (formulas from [5] are useful)

$$
\int_{0}^{\frac{\pi v}{2}} d y \ell(y)=-\frac{\pi^{2} v^{2}}{24 \cos ^{2} \frac{\pi v}{2}}(\pi v+3 \sin \pi v) .
$$

\section{COMPARISON WITH NUMERICAL RESULTS}

In this section, we compare the large $q$ result with the numerical solution of the Schwinger-Dyson equation (3). In general, we expect the large $q$ formula to work well when $|g(\tau)| \ll q$ and $|h(\tau)| \ll q^{2}$. These inequalities are fulfilled when $\beta \mathcal{J} \ll \pi e^{q / 2}$.

Looking at the explicit formula (15), it is tempting to exponentiate the result and to introduce an exponentiated large $q$ two-point function,

$$
G(\tau)=\frac{1}{2} \operatorname{sgn}(\tau) \exp \left(\frac{1}{q} g+\frac{1}{q^{2}}\left(h-\frac{1}{2} g^{2}\right)\right),
$$

which is equivalent to (5) up to order $1 / q^{2}$. We plot numerical and large $q$ results for $q=4$ and different values of $\beta J$ in Fig. 1. We can see that the exponentiated result works very precisely even for large $\beta J$, whereas the large $q$ answer (5) deviates significantly from numerics at large $\beta J$.

\section{LARGE $\boldsymbol{q}$ FREE ENERGY}

The leading large $N$ approximation to the free energy in the SYK model is $[2,6]$

$$
\begin{aligned}
-\frac{\beta F}{N}= & \log \operatorname{Pf}\left(\partial_{\tau}-\Sigma\right) \\
& -\frac{1}{2} \int_{0}^{\beta} d \tau_{1} d \tau_{2}\left(\Sigma\left(\tau_{12}\right) G\left(\tau_{12}\right)-\frac{J^{2}}{q} G\left(\tau_{12}\right)^{q}\right) .
\end{aligned}
$$

To avoid evaluating the Pfaffian it is convenient to differentiate the free energy by $J \partial_{J}[3]$

$$
\begin{aligned}
J \partial_{J}(-\beta F / N) & =-\left.\frac{\beta}{q} \partial_{\tau} G\right|_{\tau \rightarrow+0} \\
& =\left.\frac{\pi v}{2 q}\left(\frac{1}{q} \partial_{x} g+\frac{1}{q^{2}} \partial_{x} h\right)\right|_{x \rightarrow \frac{\pi v}{2}},
\end{aligned}
$$

where from (9) and (15) we find 


$$
\begin{aligned}
\left.\partial_{x} g\right|_{x \rightarrow \frac{\pi v}{2}}= & 2 \tan \frac{\pi v}{2}, \\
\left.\partial_{x} h\right|_{x \rightarrow \frac{\pi v}{2}}= & \frac{4}{1+\frac{\pi v}{2} \tan \frac{\pi v}{2}}\left(\frac{\pi v}{2}-\tan \frac{\pi v}{2}\left(1+\frac{\pi v}{2} \tan \frac{\pi v}{2}\right)\right. \\
& \left.-\int_{0}^{\frac{\pi v}{2}} d y \ell(y)\right) .
\end{aligned}
$$

Next, using (16) and

$$
J \partial_{J}=\frac{v \partial_{v}}{1+\frac{\pi v}{2} \tan \frac{\pi v}{2}},
$$

we can integrate back and obtain $-\beta F / N=\frac{1}{2} \log 2+$ $\frac{1}{q^{2}} F_{1 / q^{2}}+\frac{1}{q^{3}} F_{1 / q^{3}}+\cdots$, where

$$
\begin{aligned}
& F_{1 / q^{2}}(v)=\pi v\left(\tan \frac{\pi v}{2}-\frac{\pi v}{4}\right), \\
& F_{1 / q^{3}}(v)=\pi v\left(\pi v-2 \tan \frac{\pi v}{2}\left(1-\frac{\pi^{2} v^{2}}{12}\right)\right) .
\end{aligned}
$$

Expanding the free energy at strong coupling by using that

$$
v=1-\frac{2}{\beta \mathcal{J}}+\frac{4}{(\beta \mathcal{J})^{2}}-\frac{\left(24+\pi^{2}\right)}{3(\beta \mathcal{J})^{3}}+\cdots,
$$

we find

$$
\begin{aligned}
-\frac{\beta F}{N}= & \beta \mathcal{J}\left(\frac{1}{q^{2}}-\frac{12-\pi^{2}}{6 q^{3}}\right)+\left(\frac{1}{2} \log 2-\frac{\pi^{2}}{4 q^{2}}+\frac{\pi^{2}}{3 q^{3}}\right) \\
& +\frac{1}{\beta \mathcal{J}}\left(\frac{\pi^{2}}{2 q^{2}}-\frac{\pi^{2}\left(\pi^{2}+12\right)}{12 q^{3}}\right) \\
& +\frac{1}{(\beta \mathcal{J})^{2}}\left(-\frac{\pi^{2}}{q^{2}}+\frac{\pi^{2}\left(5 \pi^{2}+24\right)}{9 q^{3}}\right)+\cdots,
\end{aligned}
$$

where the first three terms are the ground state energy, the zero-temperature entropy and the temperature dependent correction to the entropy. The zero temperature entropy coincides with the large $q$ expansion of the formula [2,7]

$$
\frac{S_{0}}{N}=\frac{1}{2} \log 2-\int_{0}^{1 / q} d x \pi\left(\frac{1}{2}-x\right) \tan \pi x .
$$

The last term in (24) agrees with the formula reported in $[8-10]$.

Using the result (24), one can find the coefficient of the Schwarzian action. The Schwarzian action, which governs the low-energy dynamics of the SYK model is given by the formula [3,11-14]

$$
S=-N \frac{\alpha_{S}}{\mathcal{J}} \int d \tau\{f, \tau\}, \quad\{f, \tau\} \equiv \frac{f^{\prime \prime \prime}}{f^{\prime}}-\frac{3}{2}\left(\frac{f^{\prime \prime}}{f^{\prime}}\right)^{2},
$$
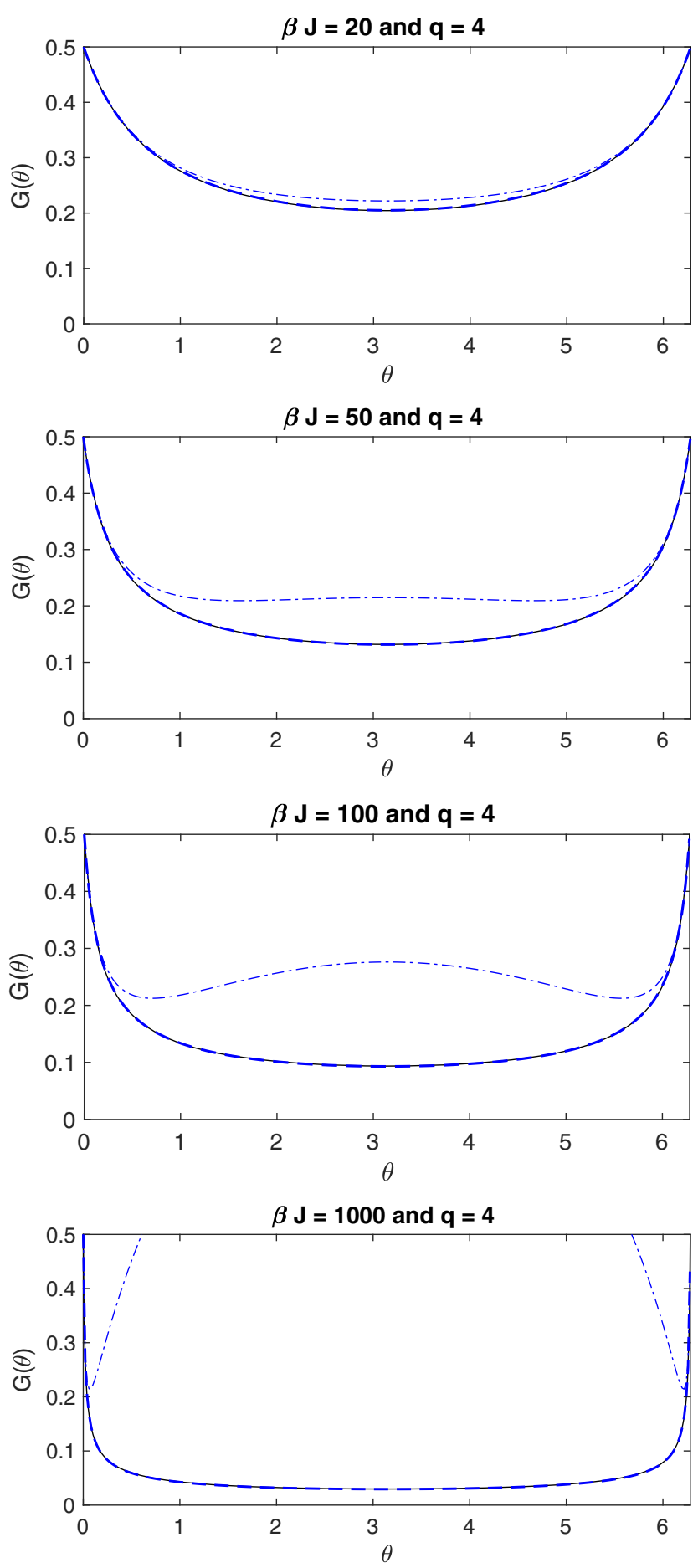

FIG. 1. Plots of the numerical solution and the large $q$ approximations for $G(\theta), \theta=2 \pi \tau / \beta$ at $\beta J=20,50,100$, 1000 and $q=4$. The black solid line is the numerical solution for the Schwinger-Dyson equation (3). The blue dash-dotted line is the large $q$ approximation (5) with $1 / q^{2}$ term. The blue dashed line is the exponentiated two-point function (17).

where the coefficient $\alpha_{S}$ depends on $q$. This coefficient is related to the finite temperature correction to the free energy, so at large $q$ using (24) we find 


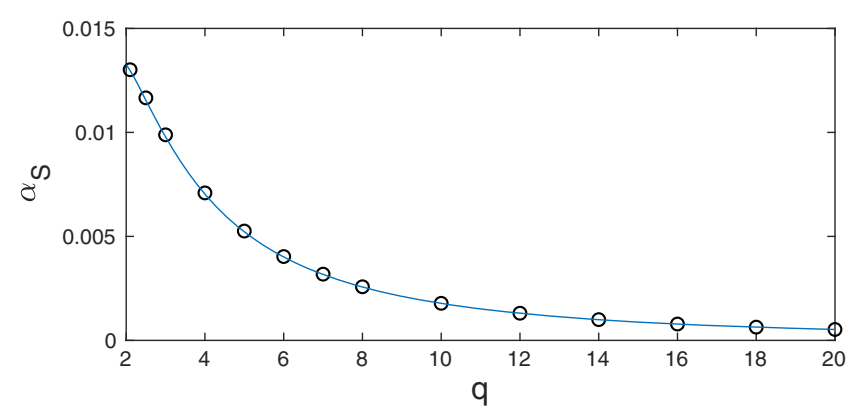

FIG. 2. Plot of $\alpha_{S}$ as a function of $q$. The black circles correspond to numerical results adapted from [3]. The blue solid line corresponds to the two-sided Pade approximation (29).

$-\frac{\beta F}{N} \supset \frac{2 \pi^{2} \alpha_{S}}{\beta \mathcal{J}}=\frac{1}{\beta \mathcal{J}}\left(\frac{\pi^{2}}{2 q^{2}}-\frac{\pi^{2}\left(\pi^{2}+12\right)}{12 q^{3}}+\cdots\right)$.

At $q=2$, one has $\alpha_{S}=\frac{1}{24 \pi}$. Using asymptotics for $\alpha_{S}$ at $q=\infty$ and $q=2$,

$$
\alpha_{S}(q)= \begin{cases}\frac{1}{24 \pi}+\cdots, & q \rightarrow 2 \\ \frac{1}{4 q^{2}}-\frac{\pi^{2}+12}{24 q^{3}}+\cdots, & q \rightarrow \infty\end{cases}
$$

we obtain two-sided Pade approximant:

$\operatorname{Pade}_{[3,1]}: \alpha_{S}(q)=\frac{\pi^{2}-18 \pi+24+3(3 \pi-2) q}{6 q^{2}\left(\pi^{3}+8+2(3 \pi-2) q\right)}$.

We note that one can improve approximation by using more terms near $q=2$ [9]. We plotted Pade approximation and numerical results adapted from [3] in Fig. 2. We see that the Pade approximation is very close to numerics.

At large $\beta \mathcal{J}$, it is possible to compute correction to the conformal propagator [3]

$\frac{\delta G}{G_{c}}=-\frac{\alpha_{G}(q)}{\beta \mathcal{J}} f_{0}\left(\frac{2 \pi \tau}{\beta}\right), \quad f_{0}(x) \equiv 2+\frac{\pi-|x|}{\tan \frac{|x|}{2}}$, where $\alpha_{G}(q)$ is some numerical coefficient that depends on $q$. Using the large $q$ result, we find

$$
\alpha_{G}(q)=\frac{2}{q}-\frac{12+7 \pi^{2}}{9 q^{2}}+\cdots
$$

\section{CONCLUSIONS}

It would be interesting to generalize the result of this article to other SYK-type models, discussed in [15-18]. In particular, it would be interesting to compute the thermalization time using the large $q$ solution for the SYK models discussed in [19].

It is also interesting to develop the $1 / q$ expansion for the higher-dimensional SYK models [20-24], where the stability of the large $N$ limit is unclear.

The large $q$ approximation to the two-point function can be used as well in studying tensor models [25-27]. Even though the general $q$ melonic tensor interactions have some ambiguities [27,28], one can formally consider the large $q$ generalization of the Schwinger-Dyson equation.

The discussion of this paper is focused on the leading terms of the large $N$ limit. However, the large $q$ expansion is also expected to be useful for the study of subleading $1 / N$ contributions $[29,30]$.

\section{ACKNOWLEDGMENTS}

G. T. would like to thank Yingfei Gu, Igor Klebanov, Subir Sachdev and Douglas Stanford for useful comments. G. T. also thanks Douglas Stanford for providing numerical results for $\alpha_{S}$ from [3]. This research was supported by the Multidisciplinary University Research Initiatives (MURI) Grant No. W911NF-14-1-0003 from Army Research Office (ARO) and by U.S. Department of Energy Grant No. de-sc0007870.
[1] S. Sachdev and J. Ye, Phys. Rev. Lett. 70, 3339 (1993).

[2] A. Kitaev, A simple model of quantum holography, http:// online.kitp.ucsb.edu/online/entangled15/kitaev/.

[3] J. Maldacena and D. Stanford, Phys. Rev. D 94, 106002 (2016).

[4] O. Parcollet and A. Georges, Phys. Rev. B 59, 5341 (1999).

[5] A. I. Davydychev and M. Yu. Kalmykov, Nucl. Phys. B699, 3 (2004).

[6] S. Sachdev, Phys. Rev. X 5, 041025 (2015).
[7] A. Georges, O. Parcollet, and S. Sachdev, Phys. Rev. B 63, 134406 (2001).

[8] J. S. Cotler, G. Gur-Ari, M. Hanada, J. Polchinski, P. Saad, S. H. Shenker, D. Stanford, A. Streicher, and M. Tezuka, J. High Energy Phys. 05 (2017) 118.

[9] A. Jevicki and K. Suzuki, J. High Energy Phys. 11 (2016) 046.

[10] A. Kitaev and S. J. Suh, J. High Energy Phys. 05 (2018) 183.

[11] J. Maldacena, D. Stanford, and Z. Yang, Prog. Theor. Exp. Phys. (2016) 12C104. 
[12] J. Engelsy, T. G. Mertens, and H. Verlinde, J. High Energy Phys. 07 (2016) 139.

[13] K. Jensen, Phys. Rev. Lett. 117, 111601 (2016).

[14] A. Jevicki, K. Suzuki, and J. Yoon, J. High Energy Phys. 07 (2016) 007.

[15] D. J. Gross and V. Rosenhaus, J. High Energy Phys. 02 (2017) 093.

[16] R. A. Davison, W. Fu, A. Georges, Y. Gu, K. Jensen, and S. Sachdev, Phys. Rev. B 95, 155131 (2017).

[17] Y. Gu, X.-L. Qi, and D. Stanford, J. High Energy Phys. 05 (2017) 125.

[18] W. Fu, D. Gaiotto, J. Maldacena, and S. Sachdev, Phys. Rev. D 95, 026009 (2017); 95, 069904(A) (2017).

[19] A. Eberlein, V. Kasper, S. Sachdev, and J. Steinberg, Phys. Rev. B 96, 205123 (2017).

[20] M. Berkooz, P. Narayan, M. Rozali, and J. Simn, J. High Energy Phys. 01 (2017) 138.
[21] G. Turiaci and H. Verlinde, J. High Energy Phys. 10 (2017) 167.

[22] J. Murugan, D. Stanford, and E. Witten, J. High Energy Phys. 08 (2017) 146.

[23] S. Giombi, I. R. Klebanov, and G. Tarnopolsky, Phys. Rev. D 96, 106014 (2017).

[24] S. Prakash and R. Sinha, J. High Energy Phys. 02 (2018) 086.

[25] E. Witten, arXiv:1610.09758.

[26] R. Gurau, Nucl. Phys. B916, 386 (2017).

[27] I. R. Klebanov and G. Tarnopolsky, Phys. Rev. D 95, 046004 (2017).

[28] C. Jepsen (private communication).

[29] A. M. Garca-Garca, Y. Jia, and J. J. M. Verbaarschot, J. High Energy Phys. 04 (2018) 146.

[30] Y. Jia and J. J. M. Verbaarschot, J. High Energy Phys. 11 (2018) 031. 